\title{
Primary structure of arabinoxylans of ispaghula husk and wheat bran
}

\author{
Sandra Edwards ${ }^{1}$, Martin F. Chaplin ${ }^{1 *}$, Anne D. Blackwood ${ }^{1}$ and Peter W. Dettmar ${ }^{2}$ \\ ${ }^{1}$ South Bank University, 103 Borough Road, London SE1 0AA, UK \\ ${ }^{2}$ Reckitt Benckiser Healthcare (UK) Ltd, Dansom Lane, Kingston upon Hull HU8 7DS, UK
}

\begin{abstract}
The primary structures of ispaghula husk and wheat bran were investigated in order to determine how and why these fibres are among the most beneficial dietary fibres. To this end, the polysaccharide preparations have been subjected to enzymic hydrolysis and methylation analysis. The results have shown ispaghula husk and wheat bran to be very-highly-branched arabinoxylans consisting of linear $\beta$-D- $(1 \rightarrow 4)$-linked xylopyranose $(\mathrm{Xyl} p)$ backbones to which $\alpha$-Larabinofuranose (Araf) units are attached as side residues via $\alpha-(1 \rightarrow 3)$ and $\alpha-(1 \rightarrow 2)$ linkages. Other substituents identified as present in wheat bran include $\beta$-D-glucuronic acid attached via the $\mathrm{C}(\mathrm{O})-2$ position, and arabinose oligomers, consisting of two or more arabinofuranosyl residues linked via $1-2,1-3$, and 1-4 linkages. Ispaghula-husk arabinoxylan is more complex having additional side residues which include $\alpha$-D-glucuronopyranose $(\mathrm{GalA} p)-(1 \rightarrow 2)$-linked- $\alpha$-Lrhamnopyranose- $(1 \rightarrow 4)-\beta$-D-Xyl $p, \alpha$-D-GalA $p$ - $(1 \rightarrow 3)$-linked- $\alpha$-L-Araf-( $1 \rightarrow 4)-\beta$-D-Xyl $p$, and $\alpha$ L-Araf-( $(1 \rightarrow 3)$-linked- $\beta$-D-Xyl $p-(1 \rightarrow 4)-\beta$-D-Xyl $p$. The beneficial effects of increased faecal bulk and water-holding capacity are undoubtedly related to the structures of the arabinoxylans, with differences in their efficacy to treat various functional bowel disorders due to their specific structural features.
\end{abstract}

Dietary fibre: Arabinoxylan: Ispaghula husk: Wheat bran

Arabinoxylans are natural polysaccharides found in the bran of grasses (Graminiae) as the major fraction of the cereal cell polysaccharides of wheat, rye, barley, oats, rice and ispaghula husk (Izydorczyk \& Biliaderis, 1995). They have highly-branched structures and form an important fraction of the NSP in dietary fibre, where they are causally linked to increased faecal bulking and water-holding capacity.

Arabinoxylans consist of linear $\beta$-D- $(1 \rightarrow 4)$-linked xylopyranose backbones to which $\alpha$-L-arabinofuranose units are attached as side residues via $\alpha-(1 \rightarrow 3)$ or $\alpha-(1 \rightarrow 2)$ linkages. Other substituents present can include $\beta$-Dglucuronic acid or its 4-O-methyl ester attached via the $\mathrm{C}(\mathrm{O})-2$ position, and ferulic acid covalently linked via an ester linkage to the $\mathrm{C}(\mathrm{O})-5$ position of arabinose. Arabinose oligomers, consisting of two or more arabinosyl residues linked via $1-2,1-3$, and 1-4 linkages, have also been reported for some arabinoxylans. Terminal galactosyl, glucosyl, and xylosyl residues can be present, but are usually quantitatively minor components and may originate, in some cases, from contaminant polysaccharides.

Most of the structural analysis in the literature is based on water-extracted arabinoxylans obtained from wheat or rye cereal grains (Bengtsson \& Aman, 1990; Hoffmann et al. 1992; Shiiba et al. 1993). The main differences between arabinoxylans concern arabinose:xylose, the relative proportions and sequences of the various linkages between these two sugars, and the presence of other substituents. Arabinose:xylose in arabinoxylans from wheat endosperm may vary from 0.5 to 0.71 but is usually lower than that found in bran (1·02-1·07; Izydorczyk \& Biliaderis, 1995). A large portion of cereal arabinoxylans cannot be extracted from the cell-wall material with water. Differences in the water extractability are related to differences in substitution patterns, the extent of physical entanglement, covalent ester bonding between carboxyl groups of uronic acids and hydroxyl groups of arabinoxylans, as well as the formation of di-ferulic acid bridges between adjacent chains (Fincher \& Stone, 1986).

Since the 1970s wheat bran and ispaghula husk have received considerable interest as sources of dietary fibre for human consumption. An important physiological effect of these NSP is faecal-bulking capacity, which is ascribed to their low degradability in the digestive tract and high water-holding capacity (Stevens \& Selvendran, 1988). The arabinoxylans appear to play the major role in this effect.

\footnotetext{
Abbreviations: IHAE, ispaghula husk extracted with alkali; IHWE, ispaghula husk extracted with water; WBAE1, wheat bran extracted with alkali; WBAE2, wheat-bran alkali extract further extracted with distilled water.

*Corresponding author: Dr Martin Chaplin, fax +44 207815 7999, email martin.chaplin@sbu.ac.uk
} 
A number of analytical techniques, including methylation analysis and specific enzymic hydrolysis, have been utilised in order to probe the nature of these two arabinoxylans to help us understand their role in the health of the gastrointestinal tract.

\section{Methods}

\section{Extraction}

Ispaghula husk (Reckitt Benckiser Healthcare Ltd, Kingston upon Hull, Humberside, UK) was extracted with either distilled water (IHWE) or $0 \cdot 1 \mathrm{M}-\mathrm{NaOH}$ (IHAE). Postdialysis the fractions were lyophilised. Wheat bran (Holland and Barrett, London, UK) was extracted using the method described previously by Bergmans et al. (1996). The extract was concentrated by vacuum evaporation and lyophilised (WBAE1). The WBAE1 was then further extracted with distilled water and lyophilised (WBAE2).

\section{Composition}

Samples were hydrolysed with 2 M-trifluoroacetic acid for $1 \mathrm{~h}$ at $121^{\circ} \mathrm{C}$. Products were analysed by high-performance anion-exchange chromatography with pulsed amperometric detection using a Dionex CarboPac PA-1 column $(250 \times 9 \mathrm{~mm})$. Protein content was determined using the Folin-Lowry protein assay (Lowry et al. 1951).

\section{Methylation analysis}

Uronic acids present in the samples were first reduced to their respective aldoses using a carbodiimide-activated reduction step (Taylor \& Conrad, 1972). The polysaccharide samples were then methylated using a modified version of the Hakomori (1964) method. The partially-methylated alditol acetates were analysed by GC-MS and quantified from the flame-ionisation detector response according to their effective C response (Sweet et al. 1975).

\section{Enzyme assays}

Arabinoxylan samples $(1 \mathrm{mg} / \mathrm{ml}$ in $50 \mathrm{mM}$-sodium acetate buffer, $\mathrm{pH} 5$ ) were incubated with a series of enzymes ( $2 \mu \mathrm{g} / \mathrm{ml}$ final concentration): arabinofuranosidases A and B (from Aspergillus awamori; Gist Brocades, Delft, Netherlands); E1 (from Aspergillus awamori; Gist Brocades) and xylanase (from Trichiderma viridei; Fluka, Poole, Dorset, UK) are endo-1,4-xylanases; Biofeed ${ }^{\circledR}$ Plus (from Humicula insolens; Novo Nordisk, A/S 2880 Bagsvaerd, Denmark) is a commercially-available enzyme preparation containing many glycosidases. Enzymes were used either singly or in various combinations and the arabinoxylan-enzyme mixtures were incubated for $24 \mathrm{~h}$ at $40^{\circ} \mathrm{C}$. The release of reducing sugars from the substrate was determined using the Nelson-Somogyi assay (Somogyi, 1952).

\section{Separation of the glycosidic digest}

Arabinoxylan oligosaccharides were prepared by incubating a solution of the arabinoxylan sample $(50 \mathrm{mg}$ in $50 \mathrm{ml}$
$0.05 \mathrm{mM}$-sodium acetate buffer, $\mathrm{pH} 5.0$ ) with the enzyme(s) $(2 \mu \mathrm{g} / \mathrm{ml})$ for a period of $24 \mathrm{~h}$ at $40^{\circ} \mathrm{C}$. The mixture was applied to a Bio-Gel P2 column (Bio-Rad, Hemel Hempstead, UK, $1000 \times 26 \mathrm{~mm})$ at $60^{\circ} \mathrm{C}$. Fractions $(1.6 \mathrm{ml})$ were eluted in distilled water and assayed for carbohydrate content using the phenol- $\mathrm{H}_{2} \mathrm{SO}_{4}$ test (Dubois et al. 1956). The column was pre-calibrated with xylose, maltose, raffinose, stachyose and blue-dextran. Appropriate fractions were pooled, concentrated and analysed for monosaccharide content.

\section{Results and discussion}

Arabinoxylans were successfully extracted from ispaghula husk and wheat bran in the following yields (\%): IHWE 89, IHAE 77·3, WBAE1 87.4, WBAE2 86.5. As the arabinoxylans are a major constituent of both dietary fibres, the yields are expectedly high. Molecular weight analysis using sizeexclusion chromatography coupled with multi-angle laser light scattering has determined that ispaghula husk has a very high molecular weight of $2.2 \times 10^{6}$ for IHWE and $1.6 \times 10^{6}$ for IHAE. In contrast, the wheat-bran molecular weights are less at approximately $1.0 \times 10^{6}$ for both extracts.

Compositionally the arabinoxylan extracts all contain similar amounts of protein $(2 \cdot 6 \%)$, which may or may not be covalently associated with the arabinoxylans. The monosaccharide composition of the arabinoxylan extracts (Table 1) shows that there are large differences between ispaghula husk and wheat bran. Ispaghula husk appears to contain many more additional sugar residues such as rhamnose and galactose and, although there are minor differences between the two ispaghula-husk extracts, it is likely that both originate from the same parent arabinoxylan. However, the two wheat-bran extracts are markedly different with one (WBAE1) having a greater arabinose:xylose value, indicating that it is more highly branched.

Of the many enzymes utilised to hydrolyse the arabinoxylan extracts those shown in Fig. 1 were the most effective. Both arabinoxylans proved to be very resistant to degradation, with wheat bran more resistant than ispaghula husk. However, after analysing the products of hydrolysis, wheat bran proved to be cleaved into a greater number of larger oligomers than ispaghula husk, which produced

Table 1. Monosaccharide composition of extracted fractions of ispaghula husk and wheat bran as determined by high-performance ion-exchange chromatography with pulsed amperometric detection*

\begin{tabular}{lccccccc}
\hline & \multicolumn{6}{c}{ Molar composition (mmol/mol total monosaccharides) } \\
\cline { 2 - 8 } Extract & Ara & Gal & GalA & Glc & GlcA & Rha & Xyl \\
\hline IHAE & 229 & 18 & 37 & 0 & 0 & 11 & 706 \\
IHWE & 201 & 17 & 44 & 0 & 0 & 19 & 719 \\
WBAE1 & 500 & 21 & 0 & 7 & 9 & 0 & 463 \\
WBAE2 & 365 & 29 & 0 & 169 & 9 & 0 & 428 \\
\hline
\end{tabular}

Ara, arabinose; Gal, galactose; GalA, galacturonic acid; Glc, glucose; GlcA, glucuronic acid; Rha, rhamnose; Xyl, xylose; IHAE, ispaghula husk extracted with alkali; IHWE, ispaghula husk extracted with water; WBAE1, wheat bran extracted with alkali; WBAE2, wheat-bran alkali extract further extracted with distilled water.

*For details of procedures, see p. 218. 


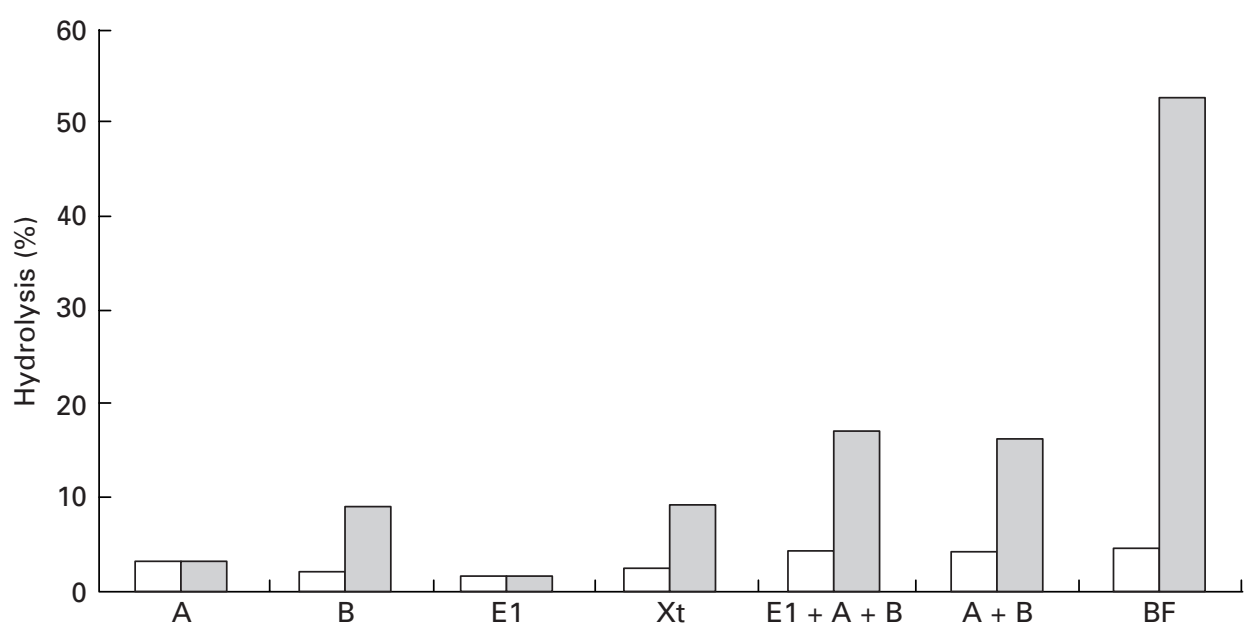

Fig. 1. Extent of enzymic hydrolysis of water-extracted ispaghula-husk ( $\mathrm{m}$ ) and alkali-extracted wheatbran $(\square)$ arabinoxylans. A, B, arabinofuranosidases from Aspergillus awamori; E1, Xt, endo-1,4xylanases from Aspergillus awamori and Trichiderma viridei respectively; BF, a commercially-available enzyme preparation containing several glycosidases. For details of enzyme preparations and procedures, see p. 218

mainly monosaccharides. However, two of the enzymes used (xylanase from $T$. viride and Biofeed ${ }^{\circledR}$ Plus) did produce small oligosaccharides from ispaghula husk. Of the singleactivity enzymes, arabinofuranosidase A produced the greater hydrolysis but, like arabinofuranosidase B, released only arabinose from the polymeric substrate (results not shown). The xylanase from $T$. viride gave the greatest hydrolysis of the four xylanases used, and released both arabinose and xylose from the main chain as well as some small oligosaccharides. The water-extractable fraction WBAE2 was much more susceptible to hydrolysis than the more highly-branched alkali-extractable WBAE1, as might have been anticipated. The enzymes were shown to work synergistically by using the arabinofuranosidases in combination with each of the xylanases.

Methylation analysis gives information on the positions at which sugar residues are substituted and their relative abundance in the polysaccharide, although it gives no direct information on their sequence or their anomeric conformation. Arabinose was linked to the xylose backbone through either the $\mathrm{C}(\mathrm{O})-3, \mathrm{C}(\mathrm{O})-2$ or $\mathrm{C}(\mathrm{O})-2,3$ position of xylose (Table 2). For wheat bran in total, only $31 \%$ of the xylose residues were unsubstituted, $24 \%$ were mono-substituted and $39 \%$ were di-substituted. The presence of 3,5-dimethyl arabinose, 2,5-dimethyl arabinose, 2,3-dimethyl arabinose and 5-methyl arabinose suggested that about $25 \%$ of the arabinose residues were present in the form of oligomeric side-chains. For the less-highly-branched WBAE2 extract, $56 \%$ of the xylose residues were unsubstituted, $21 \%$ were mono-substituted and $20 \%$ were di-substituted. The presence of both 2,4,6-trimethyl glucose and 2,3,6-trimethyl glucose suggests the presence of mixed linkage $(1 \rightarrow 3)(1 \rightarrow 4)-\beta$ glucans and possibly small amounts of $\beta$ - $(1 \rightarrow 4)$-linked glucose polymers such as cellulose. For ispaghula husk (IHAE) in total, only $20 \%$ of the xylose residues were unsubstituted, $31 \%$ were mono-substituted and $18 \%$ were di-substituted. For the IHWE extract, $18 \%$ of the xylose
Table 2. Glycosidic linkage composition of extracted fractions of ispaghula husk and wheat bran as determined by methylation analysis* $^{*}$

\begin{tabular}{lrrrr}
\hline & \multicolumn{4}{c}{ Amount (mmol/mol) $\dagger$} \\
\cline { 2 - 5 } Residues present & IHAE & IHWE & WBAE1 & WBAE2 \\
\hline t-Araf & 50 & 70 & 260 & 60 \\
1,2-linked-Araf & 0 & 0 & 10 & 6 \\
1,3-linked-Araf & 60 & 60 & 30 & 2 \\
1,5-linked-Araf & 0 & 0 & 20 & 1 \\
1,2,3-linked-Araf & 0 & 0 & 34 & 30 \\
t-Xylp & 160 & 210 & 40 & 20 \\
1,4-linked-Xylp & 40 & 30 & 200 & 460 \\
1,3-linked-Xylp & 160 & 150 & 0 & 0 \\
1,3,4-linked-Xylp & 30 & 120 & 120 & 140 \\
1,2,4-linked-Xylp & 280 & 230 & 30 & 30 \\
1,2,3,4-linked-Xylp & 180 & 110 & 250 & 170 \\
1,4-linked-GalAp & 10 & 10 & 0 & 0 \\
t-Glcp or t-GlcAp & 0 & 0 & 3 & 0 \\
1,3-linked-Glcp or -GlcAp & 0 & 0 & 0 & 8 \\
1,4-linked-Glcp or -GlcAp & 0 & 0 & 0 & 40 \\
1,2,-linked-Rhap & 10 & 10 & 0 & 0 \\
\hline
\end{tabular}

IHAE, ispaghula husk extracted with alkali; IHWE, ispaghula husk extracted with water; WBAE1, wheat bran extracted with alkali; WBAE2, wheat-bran alkali extract further extracted with distilled water; Ara, arabinose; Xyl, xylose; GalA, galacturonic acid; Glc, glucose; GlcA, glucuronic acid; Rha, rhamnose; $p$, pyranose; $f$, furanose.

* For details of procedures, see p. 218.

tCalculated as percentage of all partially-methylated alditol acetates present, based on the corrected peak area (peak area/molar response factor).

residues were unsubstituted, $31 \%$ were mono-substituted and $11 \%$ were di-substituted. The presence of 2,5-dimethyl arabinose suggests that some of the arabinose residues are present in the form of oligomeric side-chains. The presence of 2,4,6-trimethyl galactose is due to the uronic acid being converted to its native sugar, and indicates the presence of aldobiuronic acid residues, especially in the company of 
3,4-dimethyl rhamnose (Kennedy et al. 1979). The similarity between the two ispaghula-husk extracts suggests that they originate from the same parent arabinoxylan.

Enzyme hydrolysis was used in conjunction with methylation analysis. This procedure involved the use of arabinofuranosidases to digest the extracts before methylation in order to determine the positions of cleaved arabinose units on the parent arabinoxylan. This analysis confirmed that arabinose residues were present as side groups along the xylose backbone. Other more complex side groups identified in ispaghula husk are D-glucuronopyranose- $(1 \rightarrow 2)$-L-rhamnopyranose- $(1 \rightarrow 4)$-Dxylopyranose- $\beta$ - $(1 \rightarrow 4)$-xylose, $\quad$ L-arabinofuranose- $(1 \rightarrow 3)$ D-xylopyranose- $(1 \rightarrow 4)$-D-xylopyranose- $\beta$ - $(1 \rightarrow 4)$-D-xylose and D-glucuronopyranose- $(1 \rightarrow 3)$-L-arabinofuranose- $(1 \rightarrow 4)$ D-xylopyranose- $\beta$ - $(1 \rightarrow 4)$ D-xylopyranose.

The distribution of arabinosyl substituents along the xylan backbone is probably at least as important as the extent of substitution itself, since it affects the conformation and, hence, the capability of arabinoxylans to interact with each other and/or with other polysaccharides. Chain conformation and intermolecular associations have a direct bearing on the physical and functional properties of these macromolecules.

Despite the observed high extent of structural heterogeneity among cereal arabinoxylans, all recent studies point to a non-random distribution of arabinosyl residues along the xylan backbone. Although arabinoxylans are secondary gene products and as such not under strict genetic control, some of their fine structural features indicate that their biosynthesis, at least to some extent, might be controlled by enzymic specificity. The evidence suggests that during synthesis of cereal arabinoxylans the transfer of L-arabinofuranose to the growing xylan chain and/or elongation of the backbone is sterically controlled by the substitution pattern of the preceding residues.

On the basis of enzymic studies with two highly-purified endoxylanases from Aspergillus awamori, Gruppen's group (Gruppen et al. 1993) suggested the occurrence of two regions with variable substitution patterns in alkaliextractable wheat-endosperm arabinoxylan. A highlybranched region is presumed to have a rather constant structure composed mostly of repeating tetrameric units of unsubstituted and doubly-substituted xylose residues. This region was also enriched with $\mathrm{C}(\mathrm{O})-2$ mono-substituted xylose residues. A less-dense region, which was proposed to alternate, includes sequences of at least seven contiguously unsubstituted xylose residues. The differences in arabinose:xylose among alkali-extractable arabinoxylans are proposed to be due to variations in the relative proportions of these two regions.

The physiological properties of dietary fibre are dependent on the site, rate and extent of their digestion or fermentation in the gut. In the large bowel energy is salvaged from undigested carbohydrates through fermentation. Unfermented fibre can absorb water, increasing faecal bulk, resulting in the dilution of intestinal contents and lowering concentrations of putative co-carcinogens such as secondary bile acids. Furthermore, faecal bulking results in decreased colonic transit time, reducing the exposure time to irritants and co-carcinogens. Fermentation produces short- chain fatty acids, which are an important substrate for other colonic bacteria, so increasing bacterial mass. Additionally, short-chain fatty acid production reduces colonic $\mathrm{pH}$, affecting intralumen concentration of secondary bile acids by precipitation, and by inhibition of their enzymic formation from primary bile acids.

The spatial arrangement of arabinose residues is likely to affect the rate of fermentation of arabinoxylans in the gut. Terminal residues are fermented first, and terminal $\alpha$-arabinose residues are particularly susceptible to fermentation. Regions that contain less contiguous and more isolated substituted xylose residues are likely to be degraded faster and to a greater extent than regions containing more contiguous substituted xylose residues. It is clear that arabinoxylans are heterogeneous substrates containing regions that will be more or less fermentable in the gut, depending on the extent of substitution and the spatial arrangement of the residues. Retention of structure allows a gel to form, which retains many times its own weight of water and is likely to be partially dependent on the arabinose and uronic acid content of the arabinoxylans. This behaviour explains the laxation effect that wheat bran and ispaghula husk have in the gastrointestinal tract. As the arabinose residues are fermented the xylose backbone becomes exposed providing a more hydrophobic surface, which may aid in reducing the contact that potential colonic carcinogens have with the gut mucosa via a partitioning effect. Ispaghula husk is more complex and more substituted than wheat bran and therefore has a greater ability to withstand degradation and is therefore likely to be better at alleviating the symptoms of certain functional bowel disorders such as constipation. Fig. 2 shows proposed consensus structures of these wheat-bran and ispaghula-husk arabinoxylans.

\section{Acknowledgements}

The authors gratefully acknowledge the assistance of Saphwan Al Assaf of the North East Wales Institute and Mike Havler of Reckitt Benckiser Healthcare Ltd during this project. Funding towards this project was provided by Reckitt Benckiser Healthcare (UK) Ltd.

\section{References}

Bengtsson S \& Aman P (1990) Isolation and chemical characterisation of water soluble arabinoxylans in rye grain. Carbohydrate Polymers 12, 267-277.

Bergmans MEF, Beldman G, Gruppen H \& Voragen AGJ (1996) Optimisation of the selective extraction of (glucurono) arabinoxylans from wheat bran: Use of barium and calcium hydroxide solution at elevated temperatures. Journal of Cereal Science 23, 233-245.

Dubois M, Gilles KA, Hamilton JK, Rebers PA \& Smith F (1956) Colorimetric method for determination of sugars and related substances. Analytical Chemistry 28, 350-356.

Fincher GB \& Stone BA (1986) Cell walls and their components in cereal grain technology. In Advances in Cereal Science and Technology, pp. 207-295 [Y Pomeranz, editor]. St Paul, MN: American Association of Cereal Chemists Inc.

Gruppen H, Kormelink FJM \& Voragen AGJ (1993) Water un-extractable cellwall material from wheat flour. 3. A structural model for arabinoxylans. Journal of Cereal Science 18, 111-128. 

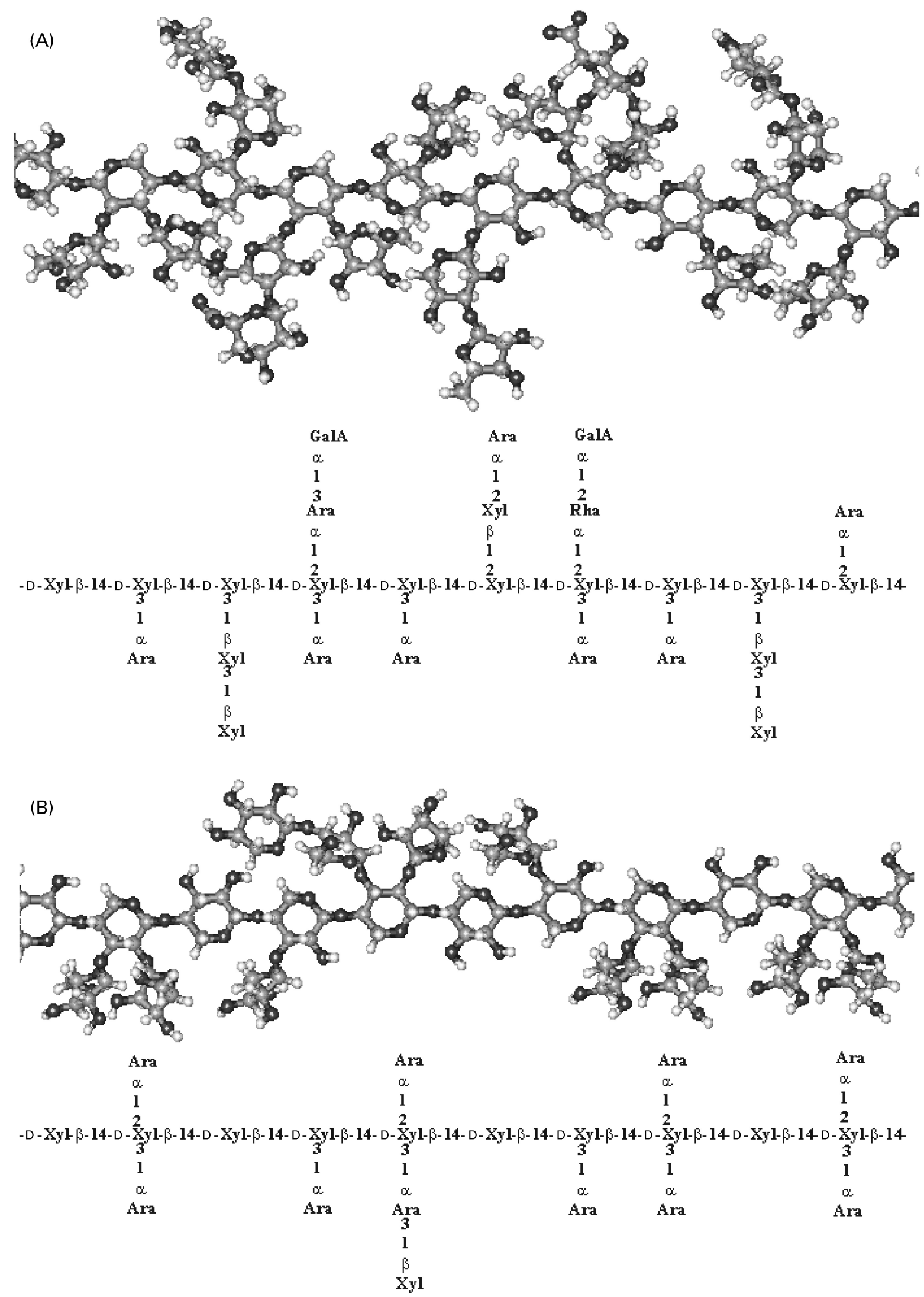

Fig. 2. Proposed consensus structures for arabinoxylans of ispaghula husk (A) and wheat bran (B). Xyl, xylose; GalA, galacturonic acid; Rha, rhamnose; Ara, arabinose. 
Hakomori S-I (1964) A rapid permethylation of glycolipids and polysaccharides catalyses by methylsulfinyl carbanion in dimethyl sulfoxide. Journal of Biochemistry (Tokyo) 55, 205-208.

Hoffmann RA, Kamerling JP \& Vliegenthart JFG (1992) Structural features of a water-soluble arabinoxylan from the endosperm of wheat. Carbohydrate Research 226, 303-311.

Izydorczyk MS \& Biliaderis CG (1995) Cereal arabinoxylans: advances in structure and physicochemical properties. Carbohydrate Polymers 28, 33-48.

Kennedy JF, Sandhu JS \& Southgate DAT (1979) Structural data for the carbohydrate of ispaghula husk ex Plantago ovata Forsk. Carbohydrate Research 75, 265-274.

Lowry OH, Rosebrough NJ, Farr AL \& Randall RJ (1951) Protein measurement with the Folin phenol reagent. Journal of Biological Chemistry 193, 265-275.
Shiiba K, Yamada H, Hara H, Okada K \& Nagao S (1993) Purification and characterisation of two arabinoxylans from wheat bran. Cereal Chemistry 70, 209-214.

Somogyi MJ (1952) Determination of blood sugar. Journal of Biological Chemistry 195, 19-23.

Stevens BJH \& Selvendran RR (1988) Changes in composition and structure of wheat bran resulting from the action of human fecal bacteria in vitro. Carbohydrate Research 183, 311-319.

Sweet DP, Shapiro RH \& Albersheim P (1975) Quantitative analysis by various GLC response factor theories for partially methylated and partially ethylated alditol acetates. Carbohydrate Research 40, 217-255.

Taylor RA \& Conrad HE (1972) Stoichiometric depolymerisation of polyuronides and glucosaminoglycuronans to monosaccharides following reduction of their carbodiimide activated carboxyl group. Biochemistry 11, 1383-1388. 